ISSN 2693-2504

\title{
Legionellosis and Public Health Epidemiology - Prevention - Treatment
}

\section{EFSTATHIOS KOUTSOSTATHIS}

Kerameikos Health Center, Greece

\section{*Correspondence author}

EFSTATHIOS KOUTSOSTATHIS

Kerameikos Health Center

Greece

Submitted : 17 Feb 2021 ; Published : 21 Mar 2021

\section{Introduction}

Legionellosis is a bacterial disease caused by bacteria of the Legionella genus. In about half of the cases, it develops as a pulmonary disease, with bilateral expansion of the lungs. In terms of clinical manifestations, it is marked by high fever, accompanied by chills, abdominal pain and diarrhoea. The public health significance of the disease is primarily that it has global spread and secondly that water systems, and mainly the hot water systems, air conditioning cooling tanks, humidifiers and other similar systems, are very much like receptors for the bacterium. Furthermore, the mortality rate of Legionella infection may reach $40 \%$ in hospitalized patients, or even higher in immunodeficient and immunosuppressed patients [1].

\section{Microbiology of the disease}

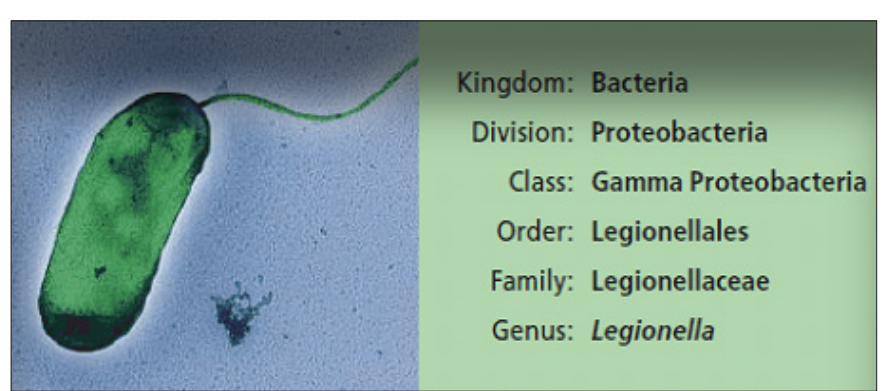

Figure 1: Legionella pneumophila, Philadelphia, USA, epidemic, 1976, adapted from Maria Ericsson, Russel Vance

(Harvard medical school electron microscopy facility)
Legionellaceae are intracellular aerobic, Gram-negative bacilli. Apart from Legionella pneumophila, which is considered responsible for $80 \%-90 \%$ of Legionella pneumonias in people, another 35 species of Legionella have been isolated, with at least 45 serogroups, including L. micdadei, that have been associated with the human disease. With regard to Legionella pneumophila, serogroup 1 of the 18 serogroups in total is the one most commonly associated with the disease in humans $[1,2]$.

\section{Epidemiology}

The bacterium is most frequently found in fresh water and water tanks. Furthermore, it has been isolated in cooling towers and potable water distribution systems, such as swimming pools and ventilators. As a matter of fact, Legionella can survive for months in distilled water or even tap water. The microorganisms are mainly transmitted through the inhalation of droplets.

It is considered the fourth most common cause of community-acquired pneumonia (rate of 3\%-15\%). Patients with chronic pulmonary disease, smokers, the aged and immunosuppressed patients are believed to be at greater risk. The same applies for diabetics, kidney patients and cancer patients.

The incubation period ranges from 2 to 10 days, with an average of 5-6 days. Sensitivity to the disease increases with age and most cases involve people aged over 50 years. The disease is most common in men rather than women (2.5/1). It is rarely encountered in people aged under 20 years $[1,2]$. 


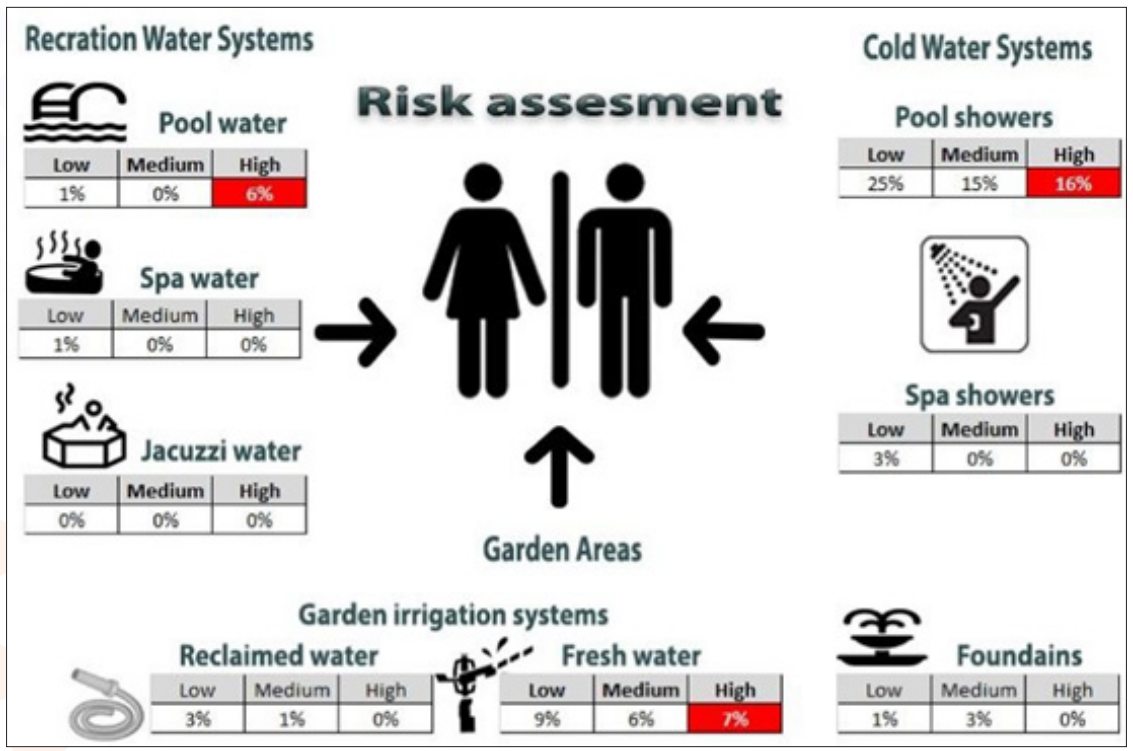

Figure 2: Risky areas associated with development of Legionella, adapted from Antonios Papadakis, Dimosthenis Chochlakis, Vassilios Sandalakis, Maria Keramarou, Yannis Tselentis, Anna Psaroulaki "Legionella spp. risk assessment in recreational and garden areas of hotels", International Journal of Environment Research and Public Health, March 2018

\section{Signs and Symptoms / Clinical Presentation}

Clinically, the disease manifests either as Pontiac fever, which has characteristics of influenza-like illness, without necessarily developing into pneumonia, or as Legionnaires' disease, which is a very severe form of atypical pneumonia and is the most common cause of admission of a patient to the ICU [2].

Legionnaires' disease, i.e. infection by Legionella, belongs to the so-called atypical pneumonias. However, many of the clinical findings for legionellosis are those of typical pneumonia, i.e. high fever, pleuritic chest pain and possible pleural effusion, coughing with sputum production and a general presentation of severe toxic pneumonia [3].

\section{Laboratory findings}

Typical laboratory findings include hyponatraemia, transaminasemia, and mild or even severe rhabdomyolysis (increased CK levels). The most sensitive method for legionellosis diagnosis is cultivation of blood mainly, as well as sputum, pulmonary tissue, if possible, or even pleural fluid in agar. The detection of the Legionella antigen in urine is a less sensitive method [3].

\section{Treatment}

Azithromycin (500 mg p.os daily), clarithromycin (500 mg p.os twice daily) and fluoroquinolones, e.g. levofloxacin (750 mg p.os once daily), constitute suitable treatment. Treatment duration is 10-14 days, while for immunosuppressed patients, 21- day treatment is recommended. These antibacterial agents present excellent intracellular concentration, as well as desired pharmacokinetic properties, and that is why they are recommended for the disease [3].

\section{Legionellosis and public health}

The significance of legionellosis, and specifically Legionnaires' disease, stems from the fact that it is a very severe type of atypical pneumonia with a mortality rate of around $40 \%$ if left untreated (with treatment, this rate drops to $0 \%-11 \%$, depending on the personal medical history of the patients). The rate increases in immunosuppressed patients (around 80\%). Furthermore, it is a disease with global spread. The first confirmed epidemic outbreak was recorded in 1957 in the USA. Since then, sporadic cases and microepidemics have been occurring in various parts of the world during all 4 seasons of the year, peaking in summer and autumn. Most Legionella-associated pneumonias develop in guests of hotels with central air-conditioning systems. Specifically, $0.5 \%-5 \%$ of community-acquired pneumonias are caused by Legionella, while it is estimated that the attack rate of epidemic outbreaks is $0.1 \%$ $5 \%$ of the general population $[1,2]$.

It is a fact that not enough statistics are collected on Legionnaires' disease on a European level. This is due to the inadequate network of case recordings in the various European Union member states. Based on the recorded evidence, around $20 \%$ is associated with travellers, either for leisure or business (travel-associated Legionnaires' disease, TALD). Italy seems to present the most travel-associated cases. Since 2005, around 5,000-6,000 cases of Legionnaires' disease have been reported in the $27 \mathrm{EU}$ member states. Based on these epidemiological data, the annual mortality rate is around $10 \%$. Interestingly enough, the epidemiological characteristics do not seem to differ much in the USA. However, it is believed that, on the one hand, the actual legionellosis cases are underdiagnosed and, on the other, they are not systematically recorded, resulting in only 1 in 10 actual cases being recorded! [4].

As far back as 2004, the World Health Organization has been implementing a Water Safety Plan, based on the Guidelines for Drinking Water Quality recommended by WHO. European Standard EN 15975-2 and Commission Directive (EU) 2015/1787 amending Council Directive 98/83/EC specify the quality of potable water and the quality of water intended for human consumption in general [5]. 


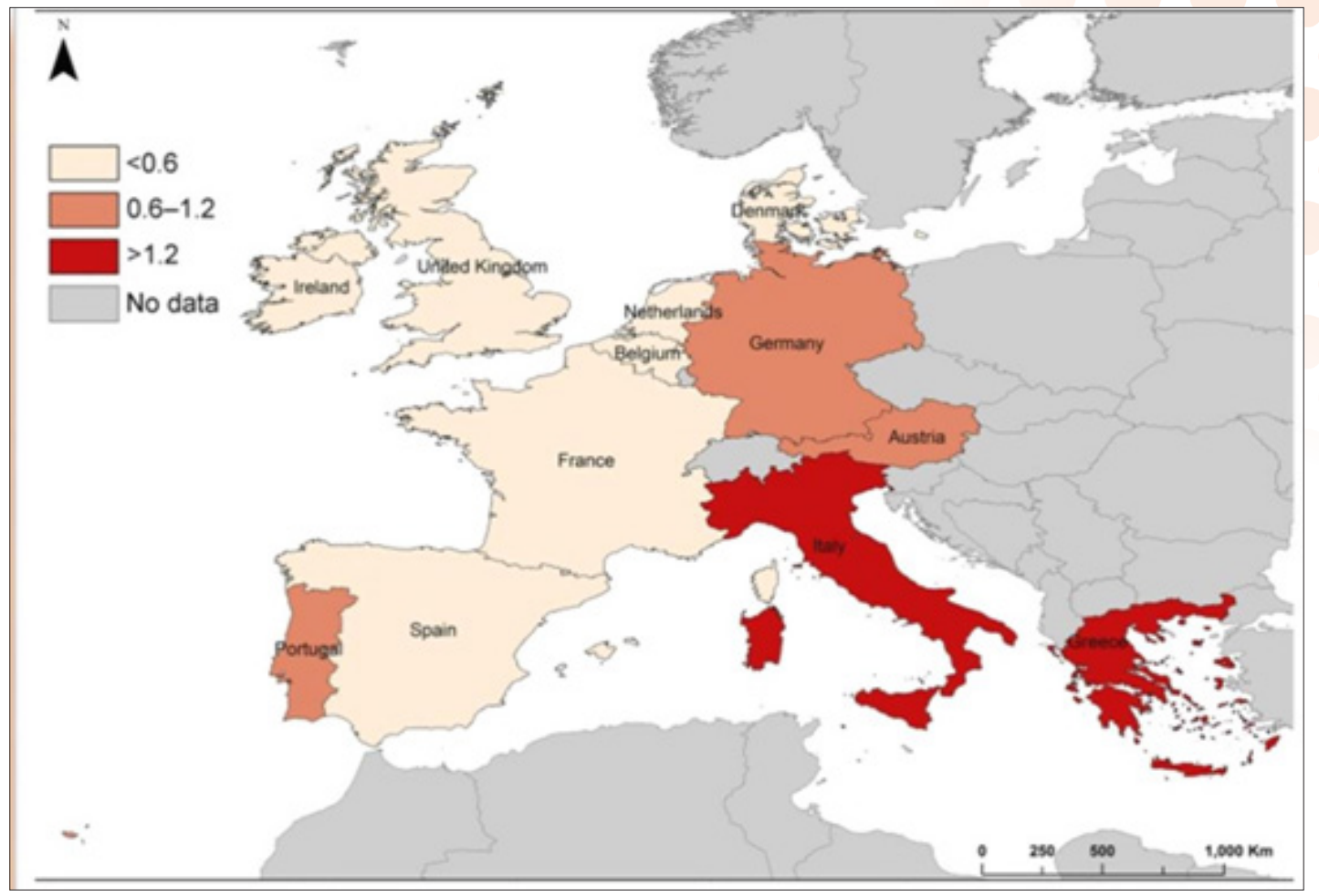

Figure 3: Risk for travel-associated legionellosis, adapted from Julien Beauté, Phillip Zucs, Birgitta de Jong "Risk for Travel -associated Legionnaires' Disease, Europe, 2009”, Emerging Infectious Diseases, Vol. 18, No. 11, Nov 2012

Preventive measures

The preventive measures include:

- $\quad$ Keeping the water temperature in the hot water system at 50o C.

- Cleaning and disinfecting the central air-conditioning cooling tanks periodically. Emptying the water when not in use.

- Using sterile water in hospital ventilators and humidifiers.

- Keeping check on those exposed to identify cases and start treatment early, in the event of an epidemic outbreak.

- Finding the source of contamination in all events. Patients do not need to be placed in isolation or have their secretions disinfected. The start of antimicrobial therapy is necessary [1].

\section{References}

1. D. Trichopoulou (2001) Proliptiki Iatriki kai Dimosia Ygeia (Preventive Medicine and Public Health), pp. 329$331,2^{\text {nd }}$ edition, 2001

2. Harrison's Principles of Internal Medicine: Companion Handbook (translation into Greek), Parisianou Publications, $16^{\text {th }}$ edition

3. Maxine Papadakis, Stephen McPhee, Current Medical Diagnosis and Treatment, Lange, 2015

4. Julien Beauté, Phillip Zucs, Birgitta de Jong (2012) "Risk for Travel associated Legionnaires 'disease, Europe, 2009", Emerging infectious diseases, 18(11).

5. Antonios Papadakis, Dimosthenis Chochlakis, Vassilios Sandalakis, Maria Keramarou, Yannis Tselentis, Anna Psaroulaki (2018) "Legionella spp. Risk Assessment in Recreational and Garden Areas of Hotels", Internation- al Journal of Environment Research and Public Health, March 2018

Copyright: (C2021 EFSTATHIOS KOUTSOSTATHIS. This is an openaccess article distributed under the terms of the Creative Commons Attribution License, which permits unrestricted use, distribution, and reproduction in anymedium, provided the original author and source are credited. 